\title{
HAEMATOLOGICAL PARAMETERS OF JAPANESE QUAIL (Coturnix coturnix japonica) KEPT IN CAGES UNDER NORMAL CONDITIONS AND EXPOSED TO LONG-TIME EXPERIMENTAL HYPODYNAMY
}

\author{
D. PRAVDA, K. BODA ${ }^{1}$, J. BAUMGARTNER, P. JELINEK, P. KUČINSKÝ, M. OKRUHLICA, \\ E. PETROVSKÁ \\ Mendel University of Agriculture and Forestry, 61300 Brmo, Czech Republic, \\ 'Institute of Experimental Veterinary Medicine, 90028 Ivanka pri Dunaji, Slovak Republic
}

Received April 14, 1995

Accepted June 6, 1996

\begin{abstract}
Pravda D., K. Boda, J. Baumgartner, P. Jelínek, P. Kučinský, M. Okruhlica, E. Petrovská: Haematological Parameters of Japanese Quail (Coturnix coturnix japonica) Kept in Cages Under Normal Conditions and Exposed to Long-term Hypodynamy. Acta vet. Brno 1996, 65: 93-97.

The aim of the study was to compare the basic haematological parameters in peripheral blood of captive Japanese quail employed as model organisms for research in cosmic biology. In the first experimental series, red blood cell count (RBC), white blood cell count (WBC), haemoglobin concentration, and erythrocytic indices, i. e. mean corpuscular volume (MCV), mean corpuscular haemoglobin $(\mathrm{MCH})$, mean corpuscular haemoglobin concentration (MCHC) of 207 clinically healthy birds of both sexes was studied. The quail were kept in groups in cages under standard laboratory conditions. In the second series, 55 female individuals exposed to experimental hypodynamy ( birds suspended in a special device) for up to 90 days were used. A total of 262 blood samples were analyzed for haematological parameters, and 5629 haematological data were evaluated using variation statistics.

Analysis of the first series made possible to establish a range of physiological values of RBC and WBC counts with significant $(P<0.05)$ sex differences in RBC counts. In general, the blood of these birds showed a high oxygenating capacity and a remarkable lymphocytic character.

Haematological parameters of quail females exposed to artificial hypodynamy for 60 days remained unaffected. However, by the end of this exposure lasting for 90 days, a mild decrease occurred in some indices. It can be concluded that Japanese quail have shown an excellent adaptation ability and can serve as a suitable model organism for the study of cosmic physiology.
\end{abstract}

Japanese quail, cosmic biology, haematology, hypodynamy

Efficient use of Japanese quail (Coturnix coturnix japonica) as a model organism for simulated and real cosmic flight experiment requires a detailed study of its physiological functions, including the respiratory, circulatory and immune ones. In this type of original studies, however, even more detailed data are required than those commonly available in literature (S turkie 1986; Ca m p bell 1989 a. o.). Haematological data on Japanese quail are, to our knowledge, still rather fragmentary. The first more systematical approach was that of A twal (1964) who studied its ontogeny. Similar basic information is provided in studies by Nirm al an and R obin s on (1971). In papers by Morg an (1980) and others, again only selected haematological data are presented.

Therefore in the first part of our study, detailed haematological parameters were determined in a population of Japanese quail bred at the Institute of Biochemistry and Genetics of the Slovak Academy of Sciences. In the second part, the same parameters were studied under condition of simulated hypodynamy. Such information is of paramount importance for any later comparison with data obtained from birds flown under real microgravity conditions during a space flight. 


\section{Materials and Methods}

A population of Japanese quail bred for generations at the Institute of Biochemistry and Genetics of the Slovak Academy of Sciences was studied. A total of 25 blood samples were collected from birds of line 02 , and 182 samples were from birds of line 07 . Quail of both sexes, aged 4-5 months, i.e. sexually mature, were included in the first series of experiment. They were reared in groups in cages, fed a commercial feed mixture for growing turkey (KT) with extra vitamin a mineral supplements Roboran $\mathrm{H}$ and Plastin at a rate of 5 and $10 \mathrm{~g}$, respectively, per $1 \mathrm{~kg}$ of feed mixture. Water was provided ad libitum. The lighting regime was $14: 10 \mathrm{~h} \mathrm{~L}: \mathrm{D}$, the temperature was $20-22^{\circ} \mathrm{C}$.

The second series of the experiment was carried out using 55 female birds suspended in a special device,jacket “ that served to ensure their partial hypodynamy. The haematological parameters were determined using 55 clinically healthy female Japanese quail suspended each in a special device designed to render them hypodynamic. The birds wore the jackets for 90 days and they were placed in cages and reared as the previous group. They were blood sampled after 35, 64 and 87 days of hypodynamy, and a total of 93 blood samples were processed with 1720 haematological parameters determined.

Blood samples were taken invariably between 9.00 and $10.00 \mathrm{~h}$ from groups of 25 birds from September till November. The blood was collected after a gentle incision with a fine scalpel of the vena basilica $\mathrm{dx}$. seu sin. onto a clean watchglass (3-4 drops), filled into micropipettes and distributed into the dilution media, i.e. Drabkin solution and Natt-Herrick solution. For haematocrit, microhaematocrit capillaries were used and the values were read with aid of the commercial Czech made equipment SOMET after 18000 r.p.m. for $2 \mathrm{~min}$, and two blood smears were made of each sample. Haemoglobin $(\mathrm{Hb})$ was determined using the cyanmethaemoglobin method, RBC and WBC were counted in Bürker's chamber. From these basic data, erythrocytic indices, i.e. mean corpuscular volume (MCV), mean corpuscular haemoglobin (MHC), and mean corpuscular haemoglobin concentration (MCHC) were calculated. Differential leukocyte count was made using blood smears. Finally, the absolute leukocyte numbers were established.

Standard deviations (S.D.), standard error of the mean (S.E.M.) and coefficient of variation were calculated, and Student's t-test was used to assess the significance of differences.

\section{Results and Discussion}

Erythrocytic indices of adult Japanese quail were characterized by a relatively high haematocrit, higher haemoglobin concentration and MCV (Table 1). Their haemogram revealed a marked lymphocytic character. In males, a higher haemoglobin concentration, higher haematocrit, and higher RBC was found along with larger MCV.

These findings seem to correspond with the neurohumoral and metabolic differences of males and females found also in other avian species ( $\mathrm{G}$ a y a thri et al. 1992; Kle in 1974; Lazar et al. 1990; S ova 1971; S ova et al. 1981; S zép et al. 1976). The erythrocytic indices found in our experiment differ from those of Atwal et al. (1974) who found considerably higher values, also from those of Nirmalan and Robinson (1971).

Table 1

Indices of the hemoglobin and erythrocytes of adult Japanese Quail under normal breeding conditions

\begin{tabular}{|c|c|c|c|c|c|c|c|c|}
\hline Parameter & $\overline{\mathbf{x}}$ & $\mathrm{n}$ & $\min$ & $\max$ & $S_{\bar{X} 11}$ & $\mathrm{~S}_{\overline{\mathrm{X}} 2}$ & $\mathrm{v}_{\overline{\mathrm{x}}}$ & $\mathbf{P}$ \\
\hline $\begin{array}{l}\mathrm{Hb} \delta \\
\mathrm{Hb} \subsetneq\end{array}$ & $\begin{array}{l}136.45 \\
117.56\end{array}$ & $\begin{array}{r}104 \\
95\end{array}$ & $\begin{array}{l}78.86 \\
80.89\end{array}$ & $\begin{array}{l}179.10 \\
185.64 \\
\end{array}$ & $\begin{array}{l}16.10 \\
20.66\end{array}$ & $\begin{array}{l}1.58 \\
2.12 \\
\end{array}$ & $\begin{array}{l}11.80 \\
17.58\end{array}$ & $++53.10^{-8}$ \\
\hline $\begin{array}{l}\text { Ery }{ }^{\circ} \\
\text { Ery } \$\end{array}$ & $\begin{array}{l}3.62 \\
3.27 \\
\end{array}$ & $\begin{array}{r}108 \\
96\end{array}$ & $\begin{array}{l}1.78 \\
1.06\end{array}$ & $\begin{array}{l}4.94 \\
4.34 \\
\end{array}$ & $\begin{array}{l}0.58 \\
0.50\end{array}$ & $\begin{array}{l}0.056 \\
0.051 \\
\end{array}$ & $\begin{array}{l}16.13 \\
15.39 \\
\end{array}$ & $\stackrel{++}{7.45 .10^{-6}}$ \\
\hline $\begin{array}{l}\mathrm{Hc} \delta \\
\mathrm{Hc} \$\end{array}$ & $\begin{array}{l}0.49 \\
0.43 \\
\end{array}$ & $\begin{array}{l}122 \\
106\end{array}$ & $\begin{array}{l}0.29 \\
0.34 \\
\end{array}$ & $\begin{array}{l}0.58 \\
0.56 \\
\end{array}$ & $\begin{array}{l}0.05 \\
0.07 \\
\end{array}$ & $\begin{array}{l}0.005 \\
0.007\end{array}$ & $\begin{array}{l}10.20 \\
16.28\end{array}$ & ${ }_{3.44 .10^{-8}}^{++}$ \\
\hline $\begin{array}{l}\text { MCHC } \\
\text { MCHC }\end{array}$ & $\begin{array}{l}28.36 \\
27.65\end{array}$ & $\begin{array}{l}117 \\
104\end{array}$ & $\begin{array}{l}22.72 \\
21.07\end{array}$ & $\begin{array}{l}46.25 \\
42.53\end{array}$ & $\begin{array}{l}3.62 \\
3.80\end{array}$ & $\begin{array}{l}0.34 \\
0.36\end{array}$ & $\begin{array}{l}12.76 \\
13.74\end{array}$ & $5.16 .10^{-1}$ \\
\hline $\begin{array}{l}\mathrm{MCH} \delta \\
\mathrm{MCH} \$\end{array}$ & $\begin{array}{l}38.59 \\
37.18\end{array}$ & $\begin{array}{r}104 \\
95\end{array}$ & $\begin{array}{l}28.54 \\
25.27\end{array}$ & $\begin{array}{r}67.59 \\
104.62 \\
\end{array}$ & $\begin{array}{r}6.99 \\
10.79 \\
\end{array}$ & $\begin{array}{l}0.69 \\
1.11 \\
\end{array}$ & $\begin{array}{l}18.12 \\
29.01\end{array}$ & $2.73 .10^{-1}$ \\
\hline $\begin{array}{l}\text { MCV ठ } \\
\text { MCV }\end{array}$ & $\begin{array}{l}138.09 \\
136.12\end{array}$ & $\begin{array}{l}120 \\
106\end{array}$ & $\begin{array}{l}79.19 \\
88.24\end{array}$ & $\begin{array}{l}235.96 \\
433.96\end{array}$ & $\begin{array}{l}24.25 \\
37.77\end{array}$ & $\begin{array}{l}2.22 \\
3.67\end{array}$ & $\begin{array}{l}17.56 \\
27.75\end{array}$ & $4.23 .10^{-1}$ \\
\hline
\end{tabular}

$+\mathrm{P} \leq 0,05$

$+P \geq 0,01$ 
Similarly, differences were found when comparing the haemoglobin and haematocrit values.

The differential white blood cell count revealed a higher percentage of heterophilic granulocytes and monocytes in females (Tables 2,3), similar to data by Atwal et al.

Table 2

Indices of the white blood component of Japanese Quail under normal breeding conditions. Number of leucocytes and leucogram

\begin{tabular}{|c|c|c|c|c|c|c|c|c|}
\hline Parameter & $\overline{\mathbf{x}}$ & $\mathbf{n}$ & $\min$ & $\max$ & $s_{\overline{\mathbf{x}}_{1}}$ & $\mathrm{~s}_{\overline{\mathrm{X}}_{2}}$ & $\mathrm{v}_{\overline{\mathrm{x}}}$ & $\mathrm{P}$ \\
\hline $\begin{array}{l}\text { Leu } \delta \\
\text { Leu } \$\end{array}$ & $\begin{array}{l}23.01 \\
23.21\end{array}$ & $\begin{array}{r}108 \\
96\end{array}$ & $\begin{array}{l}3.5 \\
7.0\end{array}$ & $\begin{array}{l}52.0 \\
47.5\end{array}$ & $\begin{array}{l}8.42 \\
8.45\end{array}$ & $\begin{array}{l}0.81 \\
0.86\end{array}$ & $\begin{array}{l}36.59 \\
36.37\end{array}$ & $0.61 .10^{-1}$ \\
\hline $\begin{array}{l}\mathrm{Ne} \text {-rods } \delta \\
\mathrm{Ne} \text {-rods } \$\end{array}$ & $\begin{array}{l}1.43 \\
1.77\end{array}$ & $\begin{array}{r}108 \\
94\end{array}$ & $\begin{array}{l}0.30 \\
0.29\end{array}$ & $\begin{array}{l}4.55 \\
4.55\end{array}$ & $\begin{array}{l}0.86 \\
0.92\end{array}$ & $\begin{array}{l}0.085 \\
0.095\end{array}$ & $\begin{array}{l}56.73 \\
51.05\end{array}$ & $8.11 \cdot 10^{-3}++$ \\
\hline $\begin{array}{l}\text { Ne-seg }{ }^{*} \\
\text { Ne-seg } ?\end{array}$ & $\begin{array}{l}34.55 \\
38.50 \\
\end{array}$ & $\begin{array}{r}108 \\
94 \\
\end{array}$ & $\begin{array}{l}13.42 \\
20.31 \\
\end{array}$ & $\begin{array}{l}67.99 \\
71.93 \\
\end{array}$ & $\begin{array}{l}10.12 \\
10.49 \\
\end{array}$ & $\begin{array}{l}0.97 \\
1.08\end{array}$ & $\begin{array}{l}29.32 \\
27.24 \\
\end{array}$ & $6.31 .10^{-3}++$ \\
\hline $\begin{array}{l}\text { Eo o } \\
\text { Eo } ?\end{array}$ & $\begin{array}{l}1.27 \\
1.44\end{array}$ & $\begin{array}{r}108 \\
94\end{array}$ & $\begin{array}{l}0.29 \\
0.32\end{array}$ & $\begin{array}{l}3.58 \\
4.50\end{array}$ & $\begin{array}{l}0.77 \\
0.84\end{array}$ & $\begin{array}{l}0.077 \\
0.089\end{array}$ & $\begin{array}{l}56.44 \\
55.38\end{array}$ & $1.47 .10^{-1}$ \\
\hline $\begin{array}{l}\text { Bas } \delta \\
\text { Bas } ?\end{array}$ & $\begin{array}{l}0.42 \\
0.59 \\
\end{array}$ & $\begin{array}{r}108 \\
94 \\
\end{array}$ & $\begin{array}{l}0.28 \\
0.26 \\
\end{array}$ & $\begin{array}{l}2.89 \\
2.48 \\
\end{array}$ & $\begin{array}{l}0.48 \\
0.47 \\
\end{array}$ & $\begin{array}{l}0.057 \\
0.054 \\
\end{array}$ & $\begin{array}{l}72.33 \\
64.81 \\
\end{array}$ & $1.49 .10^{-2}+$ \\
\hline $\begin{array}{l}\text { Lym } \delta \\
\text { Lym } \$\end{array}$ & $\begin{array}{l}59.01 \\
55.83\end{array}$ & $\begin{array}{r}108 \\
94\end{array}$ & $\begin{array}{l}27.95 \\
30.00\end{array}$ & $\begin{array}{r}83.02 \\
78.29\end{array}$ & $\begin{array}{l}10.54 \\
10.98\end{array}$ & $\begin{array}{l}1.01 \\
1.13\end{array}$ & $\begin{array}{l}17.86 \\
19.66\end{array}$ & $3.76 .10^{-2}+$ \\
\hline $\begin{array}{l}\text { Mono o } \\
\text { Mono } \$\end{array}$ & $\begin{array}{l}2.59 \\
2.14\end{array}$ & $\begin{array}{r}108 \\
94\end{array}$ & $\begin{array}{l}0.33 \\
0.32\end{array}$ & $\begin{array}{l}7.21 \\
6.28\end{array}$ & $\begin{array}{l}1.20 \\
1.14\end{array}$ & $\begin{array}{l}0.12 \\
0.12\end{array}$ & $\begin{array}{l}46.41 \\
52.33\end{array}$ & $6.89 .10^{-3}++$ \\
\hline
\end{tabular}

$+\mathrm{P} \leq 0,05$

$++P \geq 0,01$

Table 3

Absolute numbers of the individual leucocyte types of Japanese Quail under normal breeding conditions

\begin{tabular}{|c|c|c|c|c|c|c|}
\hline Parameter & $\overline{\mathbf{x}}$ & $\mathrm{n}$ & $\min$ & $\max$ & $S_{\overline{\mathbf{x}} 1}$ & $S_{\overline{\mathrm{X}} 2}$ \\
\hline $\begin{array}{l}\text { Ne-rods } \delta \\
\text { Ne-rods } \$\end{array}$ & $\begin{array}{l}0.329 \\
0.411\end{array}$ & $\begin{array}{r}108 \\
94\end{array}$ & $\begin{array}{l}0.009 \\
0.067\end{array}$ & $\begin{array}{l}1.047 \\
1.056\end{array}$ & $\begin{array}{l}0.17 \\
0.32\end{array}$ & $\begin{array}{l}0.016 \\
0.033\end{array}$ \\
\hline $\begin{array}{l}\mathrm{Ne} \text {-segm } \delta \\
\mathrm{Ne} \text {-segm } \$\end{array}$ & $\begin{array}{l}7.949 \\
8.936\end{array}$ & $\begin{array}{r}108 \\
94\end{array}$ & $\begin{array}{l}3.087 \\
4.714\end{array}$ & $\begin{array}{l}15.644 \\
16.695\end{array}$ & $\begin{array}{l}2.23 \\
2.43\end{array}$ & $\begin{array}{l}0.215 \\
0.251\end{array}$ \\
\hline $\begin{array}{l}\text { Eo } \delta \\
\text { Eo } \$\end{array}$ & $\begin{array}{l}0.292 \\
0.334\end{array}$ & $\begin{array}{r}108 \\
94\end{array}$ & $\begin{array}{l}0.067 \\
0.074\end{array}$ & $\begin{array}{l}0.824 \\
1.044\end{array}$ & $\begin{array}{l}0.19 \\
0.19\end{array}$ & $\begin{array}{l}0.018 \\
0.020\end{array}$ \\
\hline $\begin{array}{l}\text { Bas } \delta \\
\text { Bas } ?\end{array}$ & $\begin{array}{l}0.097 \\
0.137\end{array}$ & $\begin{array}{r}108 \\
94\end{array}$ & $\begin{array}{l}0.064 \\
0.060\end{array}$ & $\begin{array}{l}0.665 \\
0.576\end{array}$ & $\begin{array}{l}0.12 \\
0.13\end{array}$ & $\begin{array}{l}0.012 \\
0.013\end{array}$ \\
\hline $\begin{array}{l}\text { Lymph 'ठ } \\
\text { Lymph } \$\end{array}$ & $\begin{array}{l}13.58 \\
12.96\end{array}$ & $\begin{array}{r}108 \\
94\end{array}$ & $\begin{array}{l}6.431 \\
6.963\end{array}$ & $\begin{array}{l}19.103 \\
18.171\end{array}$ & $\begin{array}{l}2.41 \\
2.54\end{array}$ & $\begin{array}{l}0.323 \\
0.262\end{array}$ \\
\hline $\begin{array}{l}\text { Mono o } \\
\text { Mono \& }\end{array}$ & $\begin{array}{l}0.596 \\
0.495\end{array}$ & $\begin{array}{r}108 \\
94\end{array}$ & $\begin{array}{l}0.076 \\
0.074\end{array}$ & $\begin{array}{l}1.659 \\
1.458\end{array}$ & $\begin{array}{l}0.29 \\
0.27\end{array}$ & $\begin{array}{l}0.028 \\
0.028\end{array}$ \\
\hline
\end{tabular}

(1964), however with no significant differences between the sexes. These authors as well as Nirmalan and Robin s on (1971) found lower WBC in mature Japanese quail cocks. In our study, lymphocytes and heterophilic granulocytes were predominant similar to findings of the above mentioned authors, as well as their sex distribution. On the other hand, A t w a l et al. (1964) found heterophilic granulocytes as a predominant white blood cell type, similar to Nirmalan and Robinson (1971) and Ernst (1971) in Colinus virginianus. Eosinophilic granulocytes were less numerous in our material than in that of the abovementioned writers. Interestingly, a striking vacuolization was detected in the cytoplasm of more than one half eosinophilic granulocytes. 
Table 4 The influence of artificial hypodynamy in days

\begin{tabular}{|c|c|c|c|c|c|c|c|c|c|c|c|c|}
\hline 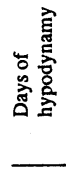 & 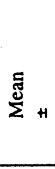 & 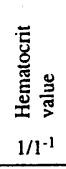 & 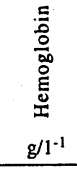 & 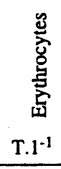 & $\begin{array}{l}3 \\
\frac{3}{2} \\
\text { f1 }\end{array}$ & $\begin{array}{l}\bar{\Xi} \\
⿱ 亠 䒑 \\
\mathrm{pg}\end{array}$ & 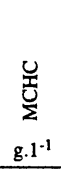 & 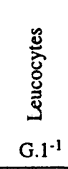 & 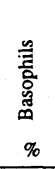 & 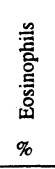 & 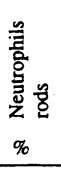 & 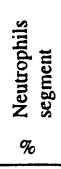 \\
\hline 0 & $\begin{array}{l}X \\
s\end{array}$ & $\begin{array}{l}0.45 \\
0.03\end{array}$ & $\begin{array}{r}116.49 \\
16.58\end{array}$ & $\begin{array}{l}3.87 \\
0.64\end{array}$ & $\begin{array}{r}121.39 \\
18.17\end{array}$ & $\begin{array}{r}32.08 \\
4.67\end{array}$ & $\begin{array}{c}25.79 \\
2.7\end{array}$ & $\begin{array}{c}16.79 \\
3.6\end{array}$ & $\begin{array}{l}0.52 \\
0.49\end{array}$ & $\begin{array}{l}3.50 \\
1.60\end{array}$ & $\begin{array}{l}0.67 \\
0.71\end{array}$ & $\begin{array}{r}24.35 \\
6.09\end{array}$ \\
\hline 35 & $\begin{array}{l}X \\
s\end{array}$ & $\begin{array}{l}0.51 \\
0.04\end{array}$ & $\begin{array}{r}131.48 \\
19.03\end{array}$ & $\begin{array}{l}4.14 \\
1.19\end{array}$ & $\begin{array}{r}127.87 \\
24.30\end{array}$ & $\begin{array}{r}32.96 \\
5.98\end{array}$ & $\begin{array}{r}26.05 \\
3.41\end{array}$ & $\begin{array}{c}27.46 \\
7.9\end{array}$ & $\begin{array}{l}0.60 \\
0.66\end{array}$ & $\begin{array}{l}3.51 \\
1.55\end{array}$ & $\begin{array}{l}0.48 \\
0.57\end{array}$ & $\begin{array}{r}19.25 \\
6.74\end{array}$ \\
\hline 64 & $\begin{array}{l}X \\
s\end{array}$ & $\begin{array}{l}0.46 \\
0.05\end{array}$ & $\begin{array}{r}127.75 \\
17.58\end{array}$ & $\begin{array}{l}4.61 \\
1.46\end{array}$ & $\begin{array}{l}97.20 \\
16.15\end{array}$ & $\begin{array}{r}26.81 \\
5.09\end{array}$ & $\begin{array}{r}27.61 \\
2.87\end{array}$ & $\begin{array}{r}12.70 \\
4.88\end{array}$ & $\begin{array}{l}0.80 \\
0.68\end{array}$ & $\begin{array}{l}4.02 \\
1.92\end{array}$ & $\begin{array}{l}0.55 \\
0.63\end{array}$ & $\begin{array}{r}30.28 \\
9.75\end{array}$ \\
\hline 87 & $\begin{array}{l}X \\
s\end{array}$ & $\begin{array}{l}0.45 \\
0.03\end{array}$ & $\begin{array}{r}117.71 \\
12.13\end{array}$ & $\begin{array}{l}4.59 \\
0.87\end{array}$ & $\begin{array}{r}101.26 \\
15.28\end{array}$ & $\begin{array}{r}26.23 \\
4.59\end{array}$ & $\begin{array}{r}25.68 \\
2.64\end{array}$ & $\begin{array}{l}9.81 \\
1.96\end{array}$ & $\begin{array}{l}0.33 \\
0.46\end{array}$ & $\begin{array}{l}2.52 \\
1.41\end{array}$ & $\begin{array}{l}0.50 \\
0.86\end{array}$ & $\begin{array}{r}27.84 \\
7.10\end{array}$ \\
\hline
\end{tabular}

Haematological parameters of female birds exposed to hypodynamy for 90 days revealed that up to day $60, \mathrm{RBC}, \mathrm{MCH}$ and $\mathrm{Hb}$ concentration tended to rise to the upper limit of the physiological range (Table 4). In the last 30 days of hypodynamy a reversed trend was observed, i.e. a decrease to the lower limit of the range established in the first series of our experiment. The WBC showed a similar shift as soon as after $\mathbf{3 0}$ days of hypodynamy, i.e. a rise and fall, however, within the physiological range. No further differences were detected in the course of the study.

Overall, the study indicates that the Japanese quail possesses an excellent adaptation capacity as far as its haematological parameters are concerned for requirements of space flights, and seems to be a suitable species for long-time exposure to cosmic life conditions.

\section{Hematologické parametry japonských křepelek (Coturnix coturnix japonica) chovaných v klecích za normálních podmínek a vystavených dlouhodobé experimentální hypodynamii}

Cílem práce bylo porovnat základní hematologické parametry v periferní krvi japonských křepelek, používaných jako modelové zvíře ve výzkumu kosmické biologie. V první sérii pokusů byly sledovány počty erytrocytů, leukocytů, koncentrace hemoglobinu a erytrocytární indexy, (střední objem erytrocytů, hemoglobin erytrocytů a střední barevná koncentrace) u 207 klinicky zdravých křepelek obou pohlaví. Křepelky byly chovány ve skupinách v klecích za standardních laboratorních podmínek. Ve druhé sérii bylo použito 55 samic vystavených experimentální hypodynamii (zavěšení ve specielním zařízení) po dobu 90 dnů. Celkem byla analyzována krev 262 jedincủ a 5629 hematologických údajủ bylo vyhodnoceno.

Analýza pruní série umožnila stanovit rozmezí fyziologických hodnot pro počty erytrocytů a leukocytů $s$ rozdíly $v$ počtech červených krvinek. Krev těchto jedinců měla vy̌̌ši oxygenační kapacitu a pozoruhodný lymfocytární charakter.

\section{Гематологические параметры японских перепелок (Coturnix coturnix japonica), содержимых в клетках с нормальными условиями и под влиянием длительной гиподинамии}

Цель работы сводилась к сопоставлению основных гематологических параметров в периферической крови японский перепелок, используемых в качестве модели в исследованиях космической биологии. В ходе первой партии опытов исследовали на 207 клинически здоровых перепелках обоих полов численность эритроцитов, лейкоцитов, 


\begin{tabular}{|c|c|c|c|c|c|c|c|c|c|c|c|}
\hline small & $\begin{array}{c}\text { Lymphocytes } \\
\text { large }\end{array}$ & both & 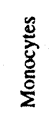 & 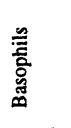 & 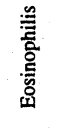 & 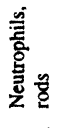 & 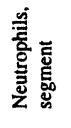 & small & $\begin{array}{c}\text { Lymphocytes } \\
\text { large }\end{array}$ & both & 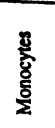 \\
\hline$\%$ & $\%$ & $\%$ & $\%$ & G.1.1 & G. $1^{-1}$ & G. $1^{-1}$ & G.1.1 & G. $1^{-1}$ & G. $1^{-1}$ & G. $1^{-1}$ & G. $1^{-1}$ \\
\hline 66.87 & 2.78 & 69.65 & 1.21 & 0.09 & 0.62 & 0.12 & 4.17 & 11.07 & 0.47 & 11.54 & 0.22 \\
\hline 7.95 & 1.57 & 7.82 & 1.28 & 0.08 & 0.38 & 0.14 & 1.57 & 2.12 & 0.29 & 2.19 & 0.24 \\
\hline 70.36 & 4.44 & 74.81 & 1.32 & 0.16 & 0.89 & 0.13 & 5.15 & 19.36 & 1.15 & 20.79 & 0.32 \\
\hline 7.36 & 2.36 & 7.50 & 0.98 & 0.20 & 0.33 & 0.14 & 1.88 & 6.78 & 0.58 & 6.87 & 0.22 \\
\hline 58.99 & 3.92 & 62.91 & 1.41 & 0.09 & 0.47 & 0.08 & 3.88 & 7.50 & 0.50 & 8.01 & 0.15 \\
\hline 10.15 & 2.55 & 10.23 & 1.55 & 0.10 & 0.18 & 0.10 & 2.11 & 3.51 & 0.39 & 3.71 & 0.14 \\
\hline 65.57 & 2.29 & 67.80 & 0.92 & 0.03 & 0.24 & 0.04 & 2.76 & 6.40 & 0.23 & 6.63 & 0.09 \\
\hline 8.55 & 1.36 & 8.43 & 0.95 & 0.04 & 0.14 & 0.07 & 1.03 & 1.29 & 0.15 & 1.35 & 0.09 \\
\hline
\end{tabular}

концентрацию гемоглобина и эритроцитарные индексы, средний объем эритроцитов. Перепелки содержались группами в клетках в стандартных лабораторных условиях. Во второй серии опытов использовали 55 самок, подверженных экспериментайьной гиподинамии (подвешенных в специальном устройстве) в течение 90 суток. В итоге проводили анализ крови 262 особей и оценивали 5629 гематологических данных.

Анализ первой серии способствовал определению пределов физиологических величин численности эритроцитов и лейкоцитов с расхождением в численности красных кровяных телец. Кровь упомянутых особей отличалась большей оксигеназной емкостью и четко выраженным лимфоцитарным характером.

Гематологические параметры самок, подвергаемых в течение 60 суток гиподинамии, остались неизменными, однако в конце эксперимента, после 90 суточной гиподинамии, наблюдали незначительное понижение некоторых индексов. Из результатов исследования вытекает, что японские перепелки отличаются исключительной адаптивной способностью и могут стать подходящей экспериментальной моделью для изучения космической физиологии.

\section{References}

ATWAL, O. S., MC FARLAND, L. Z., WILSON, W. O. 1964: Hematology of Coturnix from Birth to Maturity. Poultry Sci. 63:1392-1401

CAMPBELL, 1989: Atlas of Avian Hematology and Cytology. Iowa State University Press, 209 p.

ERNST, R. A. COLEMAN, T. H., KULENKAMP, A.W., RINGER, R. K. PANGBORN, S. 1971: The packed cell volume and differential leucocyte count of Bobwhite quail (Colinus virginianus). Poultry Sci., 50: 389

GAYATHRI, K. L., HERGE, S. N. 1992: Sex and age differences in pigeon blood. Applied zoology division, Dept. of Biosciences, Mangalore Univ. India., $574 \mathrm{p}$.

KLEIN, V. 1974: Vergleichende Haematologie, Gesamteiweissbestimmung und elektrophoretische Auftrennung im Polyamidacrylgel von Seren verschiedenen Tauben Rassen. Inaugural Diss., Tierärtztl. Fak. München, 84 p.

LAZAR, V. PRAVDA, D., STÁVKOVÁ, J. 1990: Fyziologické rozmezí hematologických hodnot hus. Živ. výr. 517-523

MORGAN, G.W. 1980: Physiological effects of exogenous adrenocorticotropin injections in Japanese Quail. Poultry Science 59: $860-867$

NIRMALAN, G. P., ROBINSON, G. A. 1971: Hematology of Japanese Quail (Coturnix coturnix japonica). Br. Poult. Sci. 52:457-482

SOVA, Z. 1971: Stanoveni základních hematologických hodnot hus chovaných v ČSSR. Project report VÚ, VŠZ Praha.

SOVA, Z., TREFNÝ, D., DOBŠINSKÁ, E., NĚMEC, Z. 1981: Jaterní biopsie, hematologické hodnoty a plazmatické lipidy při opakované intoxikaci hus Carboneum tetrachloratum. Sborník VŠZ Praha, řada B, 83-93.

STURKIE. P. D., GRIMINGER, P.: Body fluids:Blood. In: Sturkie, P.D. (Ed.) Avian Physiology, 4th Ed., Springer Verlag New York, Berlin, Heidelberg, Tokyo, pp. 102-129

SZĖP, I. 1976: Kolombozó fejtájủ Ludak néhády élertani alapertékének osskehasolíto virsgálára. Állatenjésztés 25:555. 